\title{
Raman Scattering and Efficient UV Photoluminescence from Well-Aligned ZnO Nanowires Epitaxially Grown on GaN Buffer Layer
}

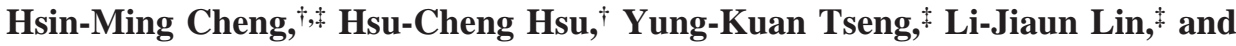 \\ Wen-Feng Hsieh*, \\ Department of Photonics and Institute of Electro-Optical Engineering, National Chiao Tung University, \\ 1001 Tahsueh Road, Hsinchu 30050, Taiwan, Republic of China, and Material Research Laboratories, \\ Industrial Technology Research Institute, Hsinchu 310, Taiwan, Republic of China
}

Received: December 15, 2004; In Final Form: February 24, 2005

\begin{abstract}
Optical phonon confinement and efficient $\mathrm{UV}$ emission of $\mathrm{ZnO}$ nanowires were investigated in use of resonant Raman scattering (RRS) and photoluminescence (PL). The high-quality $\mathrm{ZnO}$ nanowires with diameters of $80-100 \mathrm{~nm}$ and lengths of several micrometers were epitaxially grown through a simple low-pressure vaporphase deposition method at temperature $550{ }^{\circ} \mathrm{C}$ on the precoated $\mathrm{GaN}(0001)$ buffer layer. The increasing intensity ratio of $n$-order longitudinal optical (LO) phonon $\left(A_{1}(n \mathrm{LO}) / E_{1}(n \mathrm{LO})\right)$ with increasing scattering order in RRS reveals the phonon quantum confinement as shrinking the diameter of $\mathrm{ZnO}$ nanowires. The excitonrelated recombination near the band-edge transition dominate the UV emissions at room temperature as well as at low temperature that exhibits almost no other nonstoichiometric defects in the $\mathrm{ZnO}$ nanowires.
\end{abstract}

\section{Introduction}

$\mathrm{ZnO}$ is a promising material for photonic devices in the wavelength range of ultraviolet to blue since it has a wide band gap energy of $3.37 \mathrm{eV}$ and a large exciton binding energy of $60 \mathrm{meV}$ at room temperature (RT). It is expected that exciton as well as polariton lasers will be fabricated using the $\mathrm{ZnO}$ related materials and that they will overwhelm the GaN-related materials soon because excitonic-stimulated emission and optically pumped laser action in high-quality $\mathrm{ZnO}$ epitaxial films were observed ${ }^{1,2}$ at room temperature. Because of the optical losses, including not only nonradiative recombination centers but also traps of excitons, the high quality of $\mathrm{ZnO}$ becomes even more imperative in the excitonic lasing process.

Recently, one-dimensional (1D) $\mathrm{ZnO}$ nanowire has attracted considerable attention owing to its good crystal quality and unique photonic properties. ${ }^{3,4} \mathrm{ZnO}$ nanowire is also expected to play an important role as crystal quality and photonic properties interconnect as functional units in the fabrication of electronic, optoelectronic, electrochemical, and electromechanical devices with nanoscale dimensions. ${ }^{4-20}$ However, the synthesis of an array of well-aligned $\mathrm{ZnO}$ nanowires is of great interest because it is an imperative step to realize nanophotonic devices, which include light-emitting diodes and laser diodes. Different methods have been reported for fabricating arrays of well-aligned $\mathrm{ZnO}$ nanowires that include template methods, ${ }^{20,21}$ vapor transports and condensation methods, ${ }^{3,4}$ metal-organic source vapor deposition methods, ${ }^{22,23}$ and buffer layer precoated methods. ${ }^{18,24}$ The conductive $\mathrm{ZnO}$ buffer layer behaves as the nucleus for growth of wires in the meantime as the electrode for field-emission measurement. ${ }^{18}$ Park et al. ${ }^{19}$ also reported vertically aligned $n-\mathrm{ZnO}$ nanorods grown on the $p-\mathrm{GaN}$ surface. $\mathrm{GaN}$ has the same wurtzite-type structure as $\mathrm{ZnO}$ with a low

* To whom correspondence should be addressed. Tel: +886-3-5712121 ext. 56316; fax: +886-3-5716631; e-mail: wfhsieh@mail.nctu.edu.tw.

National Chiao Tung University.

$\doteqdot$ Industrial Technology Research Institute. lattice mismatch $(-1.9 \%)$. Therefore, $\mathrm{ZnO}$ nanowires on an epitaxial GaN buffer were expected to improve the crystal quality and to reduce the existence of structural defects. The $p-n$ heterojunction of $n-\mathrm{ZnO}$ and $p$-GaN showed high current density and strong electroluminescence even at a low reversed bias voltage of $3 \mathrm{~V}$. Accordingly, the buffer layer precoated method is a promising candidate for photonic device applications. This method has also been extensively used in epitaxial $\mathrm{ZnO}$ films, ${ }^{25-28}$ but it was seldom used to grow $\mathrm{ZnO}$ nanowires.

In this letter, we report that vertical arrays of well-aligned $\mathrm{ZnO}$ nanowires were epitaxially fabricated on the GaN-buffered sapphire substrate by employing catalyst-free metal vapor deposition. We will discuss the interesting optical features of phonon quantum confinement and efficient UV emission in the use of Raman and photoluminescence (PL) spectroscopy. We also confirm a very small strain in the arrays of single-crystal $\mathrm{ZnO}$ nanowires by transmission electron microscopy (TEM) and high-resolution X-ray diffraction (HRXRD).

\section{Experiments}

Synthesis of the $\mathrm{ZnO}$ nanowire arrays was carried out in a low-pressure vapor-phase deposition system, which included a horizontal alumina tube in a conventional furnace. The zinc vapor source is $99.9 \%$ pure $\mathrm{Zn}$ metal powder from Strem Chemicals. High-quality epitaxial $\mathrm{GaN}(0001)$ buffer layers of up to $2-\mu \mathrm{m}$ thick were grown on (0001) sapphire substrates by metal-organic vapor-phase epitaxy (MOVPE). The GaN/ sapphire substrates and zinc vapor source in an alumina boat were inserted into the quartz tube and placed closely to the middle of the furnace. Afterward, the chamber was heated to $550{ }^{\circ} \mathrm{C}$ at a rate of $20^{\circ} \mathrm{C} / \mathrm{min}$ under a constant flow of $55 \mathrm{sccm}$ $\mathrm{Ar}$ and $1 \mathrm{sccm}$ oxygen for $1 \mathrm{~h}$. After cooling to ambient temperature, a white deposition layer was found over the substrate. More detailed growth conditions and the description of the reaction chamber are presented elsewhere. ${ }^{24}$

The as-synthesized products were characterized by field emission scanning electron microscopy (FESEM) [LEO-1530], 
which was equipped with an energy-dispersive spectroscopy (EDS) [EDAX] facility. The crystal structure of the nanowires was analyzed using a transmission electron microscope (TEM) [JEOL JEM-2000FX]. High-resolution X-ray diffraction (HRXRD) measurements were performed in a Bede D1 diffractometer equipped with a asymmetrically cut $\mathrm{Si}(220)$ monochromator and horizontal divergence slits with a width of 100 $\mu \mathrm{m}$ and a height of $2 \mathrm{~mm}$ to select the $\mathrm{Cu} \mathrm{K}_{\alpha 1}$ radiation. An asymmetrically cut $\mathrm{Si}(220)$ analyzer crystal of $12 \operatorname{arcsec}$ acceptance permitted the separation of mosaic tilt from strain.

Conventional and resonance Raman spectroscopy were carried out by a frequency-doubled Yb:YAG laser $(\lambda=515 \mathrm{~nm})$ and a He-Cd laser $(\lambda=325 \mathrm{~nm})$, respectively, and a Jobin-Yvon T64000 microspectrometer with a 1800 grooves $/ \mathrm{mm}$ grating in the backscattering configuration was employed to investigate the lattice vibrational properties of the $\mathrm{ZnO}$ nanowires. A photoluminescence measurement was also made using a $20 \mathrm{~mW}$ $\mathrm{He}-\mathrm{Cd}$ laser at wavelength of $325 \mathrm{~nm}$, and the emitted light was dispersed by a TRIAX-320 spectrometer and detected by a UV-sensitive photomultiplier tube. A closed cycle refrigerator was used to maintain the measured temperature at $10 \mathrm{~K}$.

\section{Results and Discussion}

The general morphology of $\mathrm{ZnO}$ nanowires was obtained using field emission scanning electron microscopy (FESEM). As shown in Figure 1, vertically well-aligned arrays of $\mathrm{ZnO}$ nanowires with high density $\left(\sim 10^{10} \mathrm{~cm}^{-2}\right)$ were uniformly grown over the entire substrate. The length and diameter of the nanowires were controlled in the range between $(2.8-3.0) \mu \mathrm{m}$ and $(80-100) \mathrm{nm}$, respectively. The EDS analyses showed that the nanowires are composed of $\mathrm{Zn}$ and $\mathrm{O}$, while the element of Ga was also detected on the substrate. Further structural characterization of the $\mathrm{ZnO}$ nanowires was performed with TEM. Figure 2a displays the TEM image of a nanowire scratched from the substrate, and its selected area electron diffraction (SAED) pattern reveals the single-crystal wurtzite structure of the $\mathrm{ZnO}$ nanowire. From the high-resolution TEM (HRTEM) image in Figure 2b, we determined the lattice spacing to be $0.52 \mathrm{~nm}$ that corresponds to the $d$-spacing of (0002) crystal planes. The inset in Figure $2 b$ displays the fast Fourier transform of the HRTEM image that confirms the $\mathrm{ZnO}$ nanowires are preferentially oriented in the $c$-axis direction.

Figure 3 shows the $\theta-2 \theta$ X-ray diffraction patterns. Only the $\{0001\}$ reflection family of $\mathrm{ZnO}, \mathrm{GaN}$, and the sapphire substrate appear in the wide-angle XRD profile of Figure $3 \mathrm{a}$. Statistical evidence indicates that the epitaxial relationship between $\mathrm{ZnO}$ and $\mathrm{GaN}$ with the sapphire substrate would be $[0001]_{\mathrm{ZnO}} / /[0001]_{\mathrm{GaN}} / /[0001]_{\alpha-\mathrm{Al} 2 \mathrm{O} 3}$ along the normal of the substrate, and $[10 \overline{10}]_{\mathrm{ZnO}} / /[10 \overline{10}]_{\mathrm{GaN}} / /[11 \overline{2} 0]_{\alpha-\mathrm{Al} 2 \mathrm{O} 3}$ along the in-plane direction. More evidence of epitaxial growth was confirmed using the XRD phi-scan analysis, described in our previous report. ${ }^{24}$ Meanwhile, the measurement of XRD $\theta$-rocking curve was also performed to investigate the degree of alignment to the normal of the surface, as indicated in the inset of Figure 3a. It depicts that the $\theta$-rocking curve of $\mathrm{ZnO}(0004)$ has a full width at half-maximum (fwhm) of $0.4^{\circ}$. The fwhm of $\mathrm{ZnO}$ nanowires grown on $\mathrm{GaN}$-buffered $c$-plane sapphire was smaller than those directly grown on the $c$-plane sapphire without the buffer layer. ${ }^{22}$ The narrow fwhm of the $\mathrm{ZnO}(0004)$ diffraction peak implies that the $c$-axes of nanowires are well oriented along the normal of the substrate surface but it is still 5 times larger than the (0004) peak of GaN buffer layer because of the tilt mosaic effects on $\mathrm{ZnO}$ nanowires. Since the similar mosaic structure can be easily observed for the epi-GaN layers, ${ }^{29}$

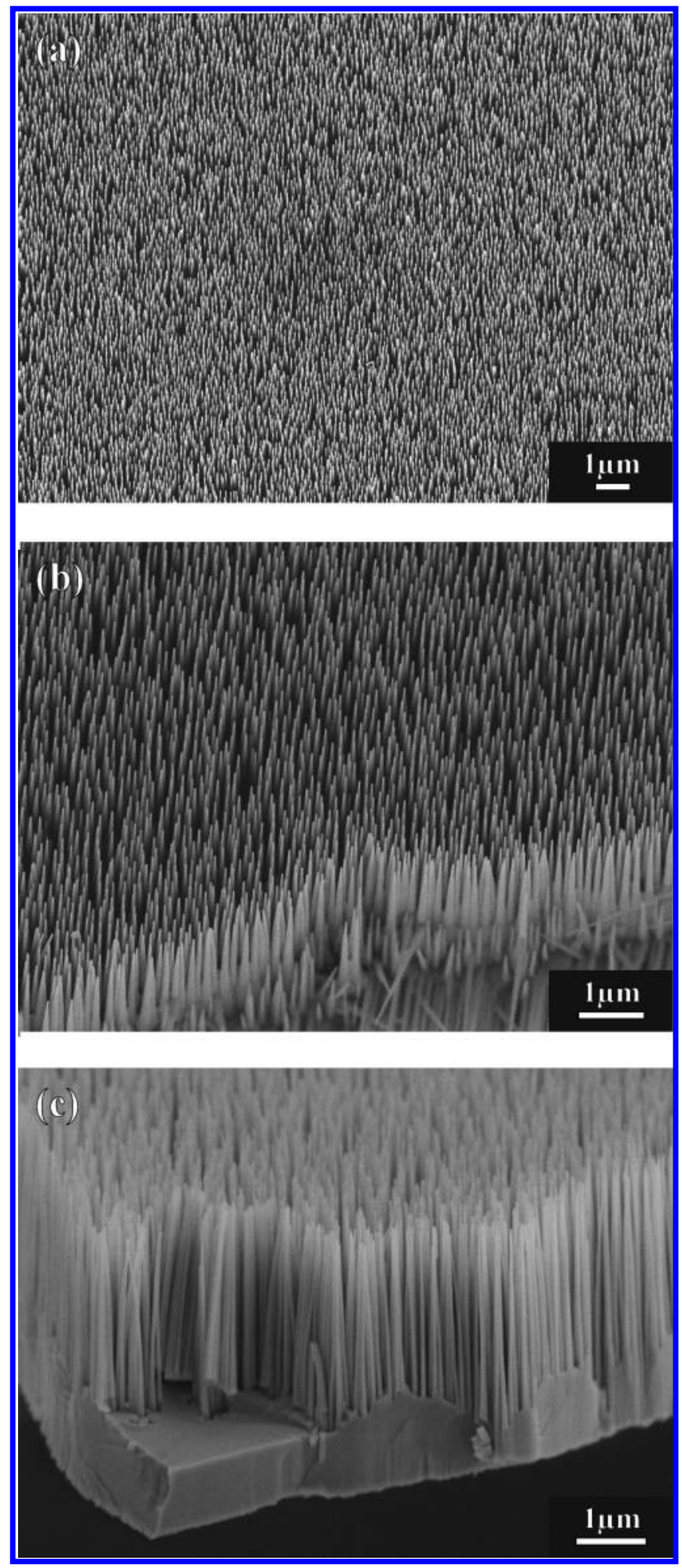

Figure 1. SEM images of vertically well-aligned $\mathrm{ZnO}$ nanowires grown on GaN-buffered sapphire substrate. (a, b) Large-scale and magnified SEM micrographs show the uniform $80-100 \mathrm{~nm}$ in diameter of $\mathrm{ZnO}$ nanowires and (c) cross-sectional image shows the length about 3.0 $\mu \mathrm{m}$.

the quality of the $\mathrm{ZnO}$ nanowires grown on epi-GaN is basically limited by the quality of epi-GaN used as the buffer layer or even worse. The HRXRD pattern shown in Figure $3 \mathrm{~b}$ demonstrates the strongly reflected $\mathrm{GaN}(0004)$ diffraction peak and the relatively weak $\mathrm{ZnO}(0004)$ peak, separately. However, the position of $\mathrm{ZnO}(0004)$ peak at $72.541^{\circ}$ corresponds to $c=$ $5.208 \AA$, which slightly differs from that of the strain-free $\mathrm{ZnO}$ 


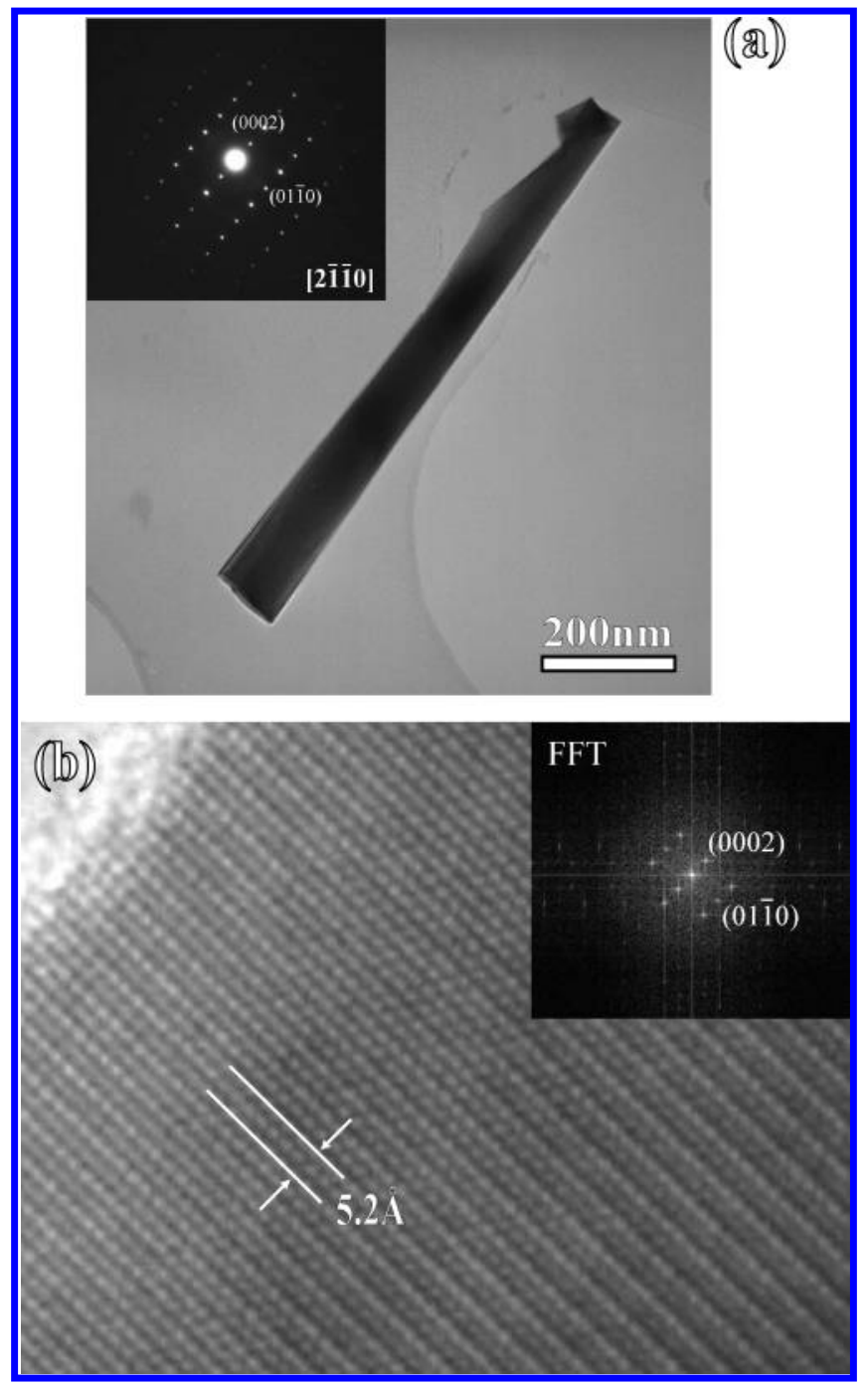

Figure 2. (a) TEM image of wurtzite structure $\mathrm{ZnO}$ nanowire and the corresponding electron diffraction pattern (inset). (b) HRTEM image with inset of its corresponding FFT pattern.

powder ${ }^{30}$ with $c=5.206 \AA$. The wurzite $\mathrm{ZnO}$ crystal would be elongated along the $c$-axis under a tensile strain in $c$-plane.

To further explore the strain in $\mathrm{ZnO}$ nanowires, reciprocal space mapping (RSM) was carried out to determine $a$ and $c$ lattice parameters of $\mathrm{ZnO}$ nanowires. Figure 4 shows the RSM of the asymmetric (202 4 ) reflection of the arrays of $\mathrm{ZnO}$ nanowires and the epi-GaN buffer layer. The reflection of the underlying $\mathrm{GaN}$ template has always been used as a reference and therefore the points in reciprocal space are given by $l$ and $k$ in units of the reciprocal vectors of $\mathrm{GaN},[0001]_{\mathrm{GaN}}$, and $[10 \overline{1} 0]_{\text {GaN }}$, respectively. For the wurtzite materials such as $\mathrm{GaN}$ and $\mathrm{ZnO}$, the magnitudes of these vectors are $|[0001]|=$ $(2 \pi / c)$ and $|[10 \overline{1} 0]|=(4 \pi / \sqrt{3} a)$, which unambiguously determine the correlation between the values of $l$ and $k$ and the lattice constants $c$ and $a$ in real space, respectively. Because the $\mathrm{GaN}$ buffer layer has $c_{\mathrm{GaN}}=5.192 \AA$ and $a_{\mathrm{GaN}}=3.188 \AA$, which is also strained by the sapphire substrate, the lattice constants of $\mathrm{ZnO}$ nanowires in the arrays can be estimated to be $c_{\mathrm{ZnO}}=$ $5.208 \AA$ and $a_{\mathrm{ZnO}}=3.248 \AA$ by use of the relationships: $l^{*} \mid$ $[0001]_{\mathrm{GaN}} \mid=(2 \pi) /\left(c_{\mathrm{ZnO}} / 4\right)$ and $h^{*}\left|[10 \overline{1} 0]_{\mathrm{GaN}}\right|=(4 \pi) /$ $\left(\sqrt{3} a_{\mathrm{ZnO}} / 2\right)$. The lattice constants slightly differ from those of the strain-free $\mathrm{ZnO}$ powder, ${ }^{30}$ which has $c_{\mathrm{o}}=5.2066 \AA$ and $a_{\mathrm{o}}$ $=3.2498 \AA$; thus, the strains $\epsilon_{z z}=\epsilon_{c c}=\left(c-c_{\mathrm{o}}\right) / c_{\mathrm{o}}>0$ and $\epsilon_{x x}$ $=\epsilon_{a a}=\left(a-a_{\mathrm{o}}\right) / a_{\mathrm{o}}<0$, indicate a biaxial compressive strain in the $a$-plane, and an uniaxial tensile strain along the $c$-axis.

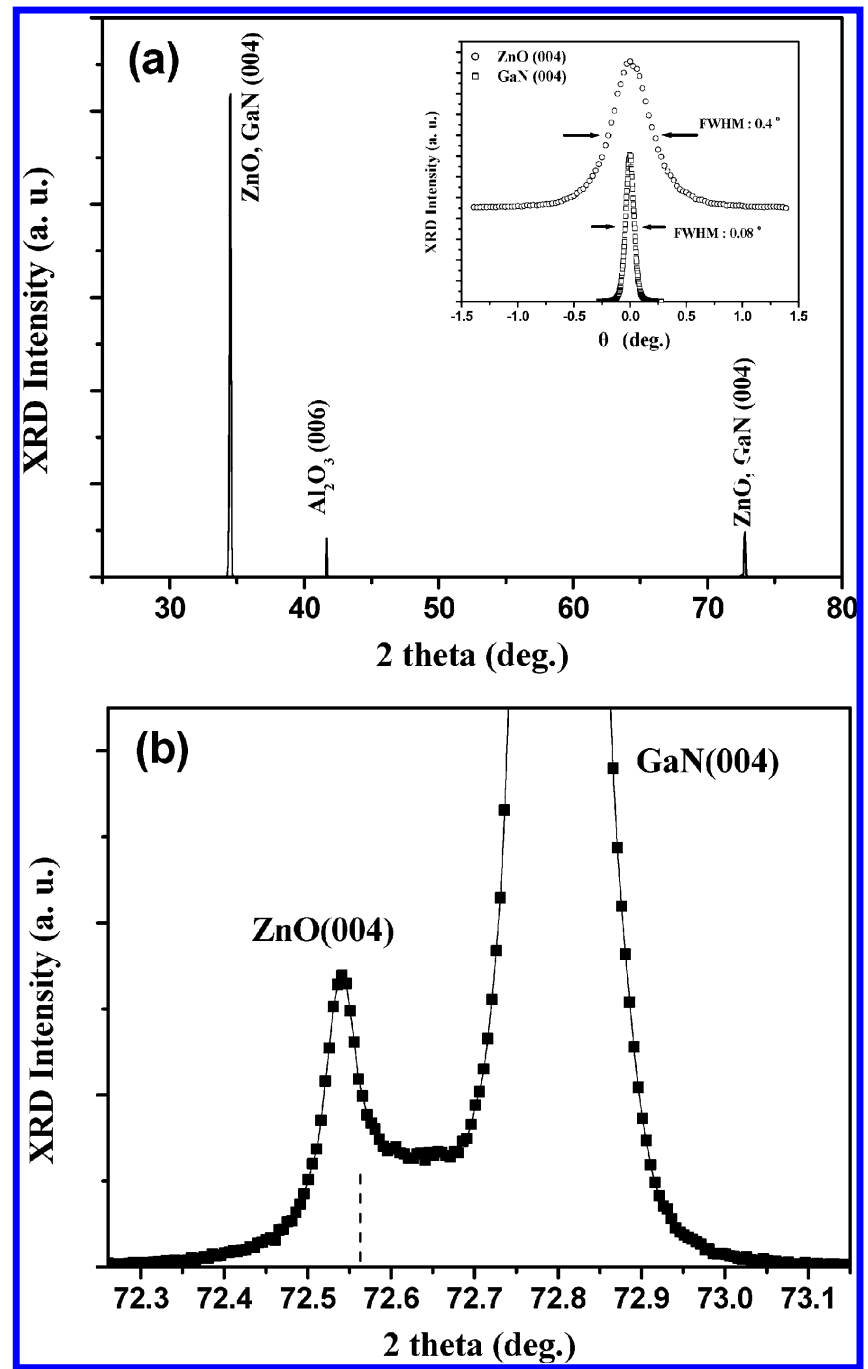

Figure 3. (a) $\theta-2 \theta$ XRD profile of $\mathrm{ZnO}$ nanowires on epi-GaN $(001) /$ $\alpha-\mathrm{Al}_{2} \mathrm{O}_{3}(001)$. The inset depicts the $\theta$-rocking curves of $\mathrm{ZnO}(004)$ and $\mathrm{GaN}(004)$, respectively. (b) HRXRD shows the (004) reflection of $\mathrm{ZnO}$ nanowires slightly shift from that of the strain-free $\mathrm{ZnO}$ powder (dash line).

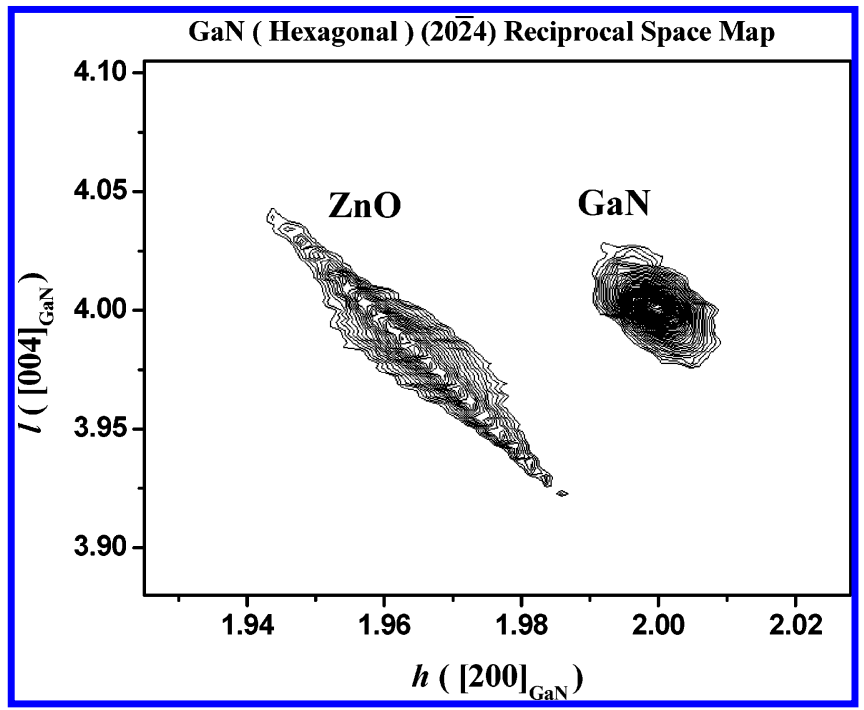

Figure 4. Reciprocal space map of asymmetric (2024) diffraction spots of a $\mathrm{ZnO}$ nanowires and epi-GaN buffer layer. The intensities are plotted logarithmically.

Since the thermal expansion coefficient of $\mathrm{ZnO}\left(6.51 \times 10^{-6} /\right.$ $\mathrm{K})$ is a little distinct from that of $\mathrm{GaN}\left(5.59 \times 10^{-6} / \mathrm{K}\right)^{28}$ and 
the reaction occurs at the low temperature of $550{ }^{\circ} \mathrm{C}$, it is expected that the strain comes from the nature lattice misfit between $\mathrm{ZnO}$ and $\mathrm{GaN}$. The residual in-plane biaxial stress can be obtained via the equation ${ }^{31}$

$$
\sigma=-453.6 \times 10^{9} \epsilon_{z z}
$$

It can thus be calculated that the arrays of $\mathrm{ZnO}$ nanowires have a compressive stress of $-121.9 \mathrm{MPa}$ when using the strain value $\epsilon_{z z}=0.0269 \%$, which is quite smaller than that of $\mathrm{ZnO}$ nanowires grown on other substrates..$^{32}$

The UV Raman scattering was performed to investigate the vibrational properties of the arrays of $\mathrm{ZnO}$ nanowires at room temperature. Since the wurtzite structure of $\mathrm{ZnO}$ and $\mathrm{GaN}$ belongs to the space group $C_{6 v}{ }^{4}\left(P_{6} \mathrm{mc}\right)$, one primitive cell includes two formula units, with all of the atoms occupying $2 b$ sites of symmetry $C_{3 v}$. Group theory predicts the existence of the following optic modes: $A_{1}+2 B_{1}+E_{1}+2 E_{2}$ at the $\Gamma$ point of the Brillouin zone; $B_{1}$ (low) and $B_{1}$ (high) modes are normally silent; $A_{1}, E_{1}$, and $E_{2}$ modes are Raman active; and $A_{1}$ and $E_{1}$ are also infrared active. Thus, $A_{1}$ and $E_{1}$ are split into longitudinal (LO) and transverse (TO) optical components. Figure 5a shows a normal Raman spectrum of the $\mathrm{ZnO}$ nanowires which was taken with an accumulation time of 300 s from a $1 \mu \mathrm{m}^{2}$ spot size excited by a frequency-doubled $\mathrm{Yb}$ : YAG laser $(\lambda=515 \mathrm{~nm})$. As we can see, the remarkable $E_{2^{-}}$ (high) mode of $\mathrm{ZnO}$ is located at $437 \mathrm{~cm}^{-1}$; the weak and almost invisible signal around $580 \mathrm{~cm}^{-1}$ is contributed to the $A_{1}(\mathrm{LO})$ and $E_{1}(\mathrm{LO})$; and the $E_{1}(\mathrm{TO}), E_{2}(\mathrm{high})$, and $A_{1}(\mathrm{LO})$ modes that belong to the epi-GaN buffer layer were also observed at 559 , 569 , and $737 \mathrm{~cm}^{-1}$, respectively, because of the penetration of 515 -nm laser light. In the backscattering geometry, the $E_{1}(\mathrm{LO})$ mode is theoretically forbidden according to the Raman selection rules, however, it can be still visible if the incident direction of the excited light beam with respect to the crystal axes is not well-defined in the use of the focusing lens for micro-Raman measurement, and thus mixed modes may be observed. Furthermore, the bending and the tilting of $\mathrm{ZnO}$ nanowires may probably cause the appearance of the $E_{1}(\mathrm{LO})$ mode.

For the advanced study, we used a $\mathrm{He}-\mathrm{Cd}$ laser $(\lambda=325$ $\mathrm{nm})$ as the excitation source. In resonant Raman scattering (RRS), the exciting photon energy is resonant with the electronic interband transition energy of the wurtzite $\mathrm{ZnO}$. The polar symmetry makes the $A_{1}(\mathrm{LO})$ and $E_{1}(\mathrm{LO})$ modes the dominant modes and it may exhibit different frequencies from the transverse optic (TO) modes. An intense multiphonon scattering was observed in the resonant Raman spectrum of Figure 5b, where four major bands were observed, centered at 577, 1154, 1729 , and $2300 \mathrm{~cm}^{-1}$, with bandwidths of $28,49,58$, and 62 $\mathrm{cm}^{-1}$, respectively, which mainly result from the polar symmetry modes $A_{1}(\mathrm{LO})$ and $E_{1}(\mathrm{LO})$ and their overtones. The first-order Raman mode at $577 \mathrm{~cm}^{-1}$ is a superposition of the $A_{1}(\mathrm{LO})$ mode at $574 \mathrm{~cm}^{-1}$ and the $E_{1}(\mathrm{LO})$ mode at $583 \mathrm{~cm}^{-1}$ in which $\mathrm{Zn}$ atoms and $\mathrm{O}$ atoms have the same vibration direction, respectively, to the neighbor lattices of the wurtzite $\mathrm{ZnO} .{ }^{33}$ The weak peak, around $437 \mathrm{~cm}^{-1}$, which is contributed to the $E_{2}$ (high) mode around $437 \mathrm{~cm}^{-1}$, is almost imperceptible as a result of other (overlapping/stronger) RRS peaks. The absence of the TO modes could be attributed to the special angle between the wave vector of photons and the $c$-axis of the wurzite $\mathrm{ZnO}$ crystals in the near backscattering geometry employed in our measurement.

Multiphonon scattering processes have also been previously reported for single crystalline bulk $\mathrm{ZnO},{ }^{34}$ and recently for $\mathrm{ZnO}$ films, ${ }^{35} \mathrm{ZnO}$-opal structures, ${ }^{36}$ and $\mathrm{ZnO}$ nanowires grown on $\mathrm{m}$-sapphire. ${ }^{37}$ The line widths of multiphonon scatterings cannot
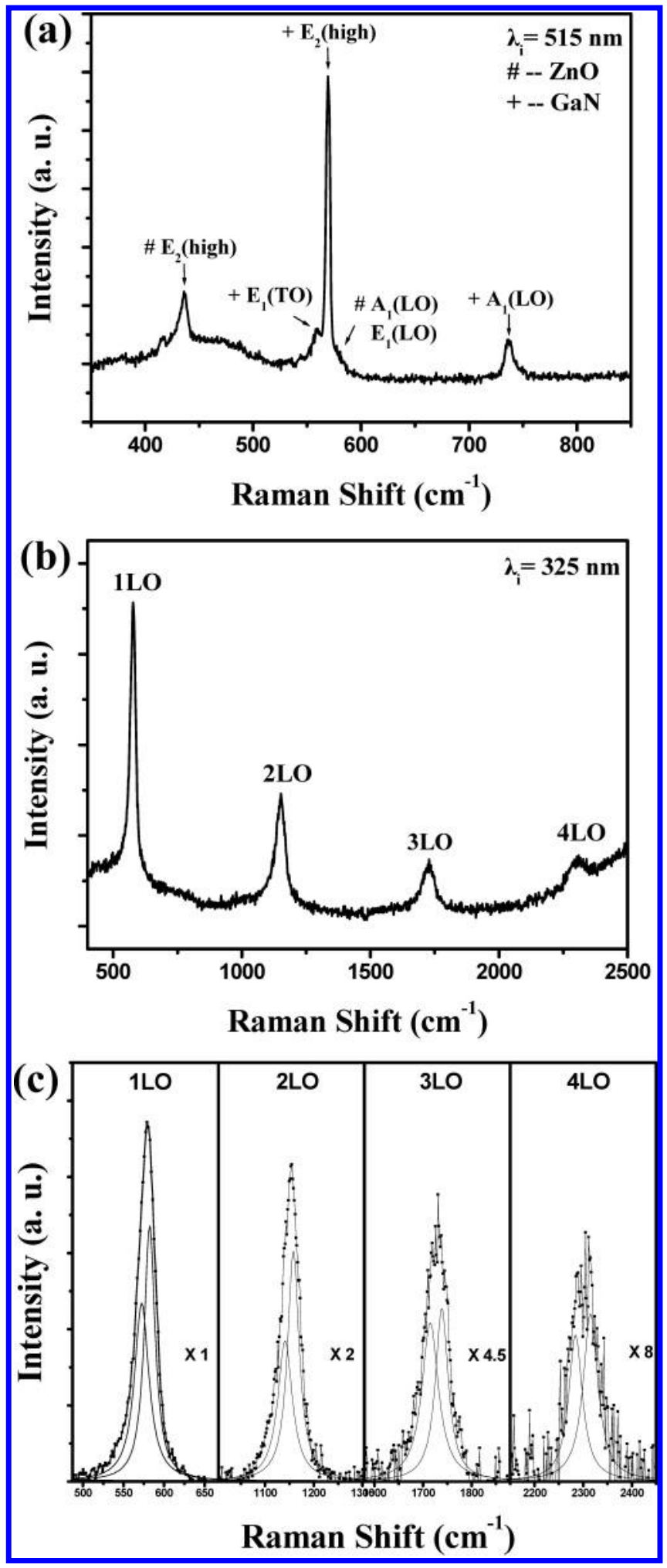

Figure 5. (a) Conventional Raman spectrum of $\mathrm{ZnO}$ nanowires on $\mathrm{GaN}$ buffer layer at room temperature using a frequency-doubled $\mathrm{Yb}$ : YAG laser $(\lambda=515 \mathrm{~nm}$ ). (b) Resonant Raman scatterings (RRS) of $\mathrm{ZnO}$ nanowires on $\mathrm{GaN}$ buffer layer using a $\mathrm{He}-\mathrm{Cd}$ laser $(\lambda=325$ $\mathrm{nm})$. The $n \mathrm{LO}$ refers to the $n$th longitudinal optical phonon. (c) Fitting profile of RRS spectrum and its decomposition. The scatter lines represent the recorded $n$ LO RRS, and the solid lines are its decomposition which indicate $A_{1}(n \mathrm{LO})$ and $E_{1}(n \mathrm{LO})$, respectively.

be explained by the equation ${ }^{34} \sigma(n \mathrm{LO})=9 n\left(\mathrm{~cm}^{-1}\right)$. The observed broadening of phonon line width is attributed to the phonon quantum confinement in $\mathrm{ZnO}$ nanowires. A similar broadening effect has also been observed in $\mathrm{ZnO}$ nanoparticles ${ }^{38}$ 
and in $\mathrm{ZnO}$ nanowires, ${ }^{37}$ which were grown on an m-sapphire substrate. Careful analysis of the resonant Raman spectrum was performed by the peak fitting, as shown in Figure 5c. The luminescence baseline was first removed and double Lorentzian profiles were chosen to fit the individual peaks of $A_{1}(n \mathrm{LO})$ and $E_{1}(n \mathrm{LO})$. The results of curve fitting give the multiphonon scattering of $A_{1}(n \mathrm{LO})$ located at 571, 1139, 1713, and 2282 $\mathrm{cm}^{-1}$, with the multiphonon scattering of $E_{1}(n \mathrm{LO})$ located at $581,1156,1737$, and $2313 \mathrm{~cm}^{-1}$. The intensity ratio of $A_{1}(n \mathrm{LO}) /$ $E_{1}(n \mathrm{LO})$ was obviously increased for the higher order phonon scattering. Because the atoms in the $E_{1}$ mode displace along the $a$-axis, they could be easily restrained with the degrees of freedom defined by the cross section of $\mathrm{ZnO}$ nanowires. On the contrary, the $A_{1}$ mode is less affected by shrinking the diameter of $\mathrm{ZnO}$ wires because of its vibration along the $c$-axis. Accordingly, the intensity ratio of $A_{1} / E_{1}$ increases as the order of phonon scattering is increased. It is again further evidence of the phonon quantum confinement in $\mathrm{ZnO}$ nanowires.

Figure 6a shows a typical PL spectrum taken at room temperature, which consists of a strong UV peak at $3.26 \mathrm{eV}$ and relatively no other visible emission. The UV band was attributed to the exciton-related emission near the band-edge, usually reported as the recombination of free excitons. The strong UV emission with no visible band in the PL spectra indicates that the $\mathrm{ZnO}$ nanowires grown on $\mathrm{GaN}$ have a good crystal quality with few nonstoichiometric defects. To investigate the UV emission in more detail, Figure $6 \mathrm{~b}$ shows the lowtemperature photoluminescence (LTPL) spectra measured at $T$ $=10 \mathrm{~K}$ under various laser intensities. The excitation intensities were set from $0.05 \mathrm{Wcm}^{-2}$ to $12 \mathrm{Wcm}^{-2}$. The bound exciton peak situated at $3.343 \mathrm{eV}$ is predominant with its longitudinal (LO) phonon replicas at the lower energy side. In the inset of Figure 6b, other than this dominant peak that has been previously reported as the recombination of excitons bound to neutral donors or acceptors, ${ }^{39,40}$ there is an additional weak feature situated on the high-energy shoulder around $3.364 \mathrm{eV}$. This higher energy emission is attributed to the donor-bound exciton $\left(\mathrm{D}^{\circ} \mathrm{X}\right)$ which is usually observed in $\mathrm{ZnO}$ single crystals, ${ }^{41}$ epitaxial layers, ${ }^{42}$ and other reports of 1-D $\mathrm{ZnO}$ rods and wires. ${ }^{20,43}$

With an increase in excitation intensity, we observed a remarkable increase in the PL intensity, with neither a blue nor a red shift. The variation of the integrated emission intensity versus the excitation laser intensity can be fitted to the simple power law: $I \propto L^{\gamma}$, as shown in Figure 6c, where $I$ is the PL intensity, $L$ is the excitation laser intensity, and $\gamma$ is a dimensionless exponent. The integrated intensity of the PL band increases superlinearly with the excitation intensity, with $\gamma=$ 1.039 and 1.015 for the 3.343 and $3.364 \mathrm{eV}$ peaks, respectively. The exponent $\gamma$ generally lies between 1 and 2 for the freeand bound-exciton emission and is less than or equal to 1 for free-to-bound and donor-acceptor pair recombinations. ${ }^{44}$ The obtained values of $\gamma=1.039$ and 1.015 confirm our assignment that the observed UV emission bands of $\mathrm{ZnO}$ nanowires are due to excitonic emissions.

\section{Conclusion}

High-density, vertically oriented arrays of $\mathrm{ZnO}$ nanowires were successfully epitaxial grown on the $\mathrm{GaN}(0001)$-buffered sapphire substrate without any catalyst. FESEM micrographs demonstrate that the $\mathrm{ZnO}$ nanowires possess a good crystalline structure with diameters of $80-100 \mathrm{~nm}$ and lengths of several micrometers. TEM micrograph and HRXRD spectra demonstrate that the arrays of $\mathrm{ZnO}$ nanowires are well aligned along
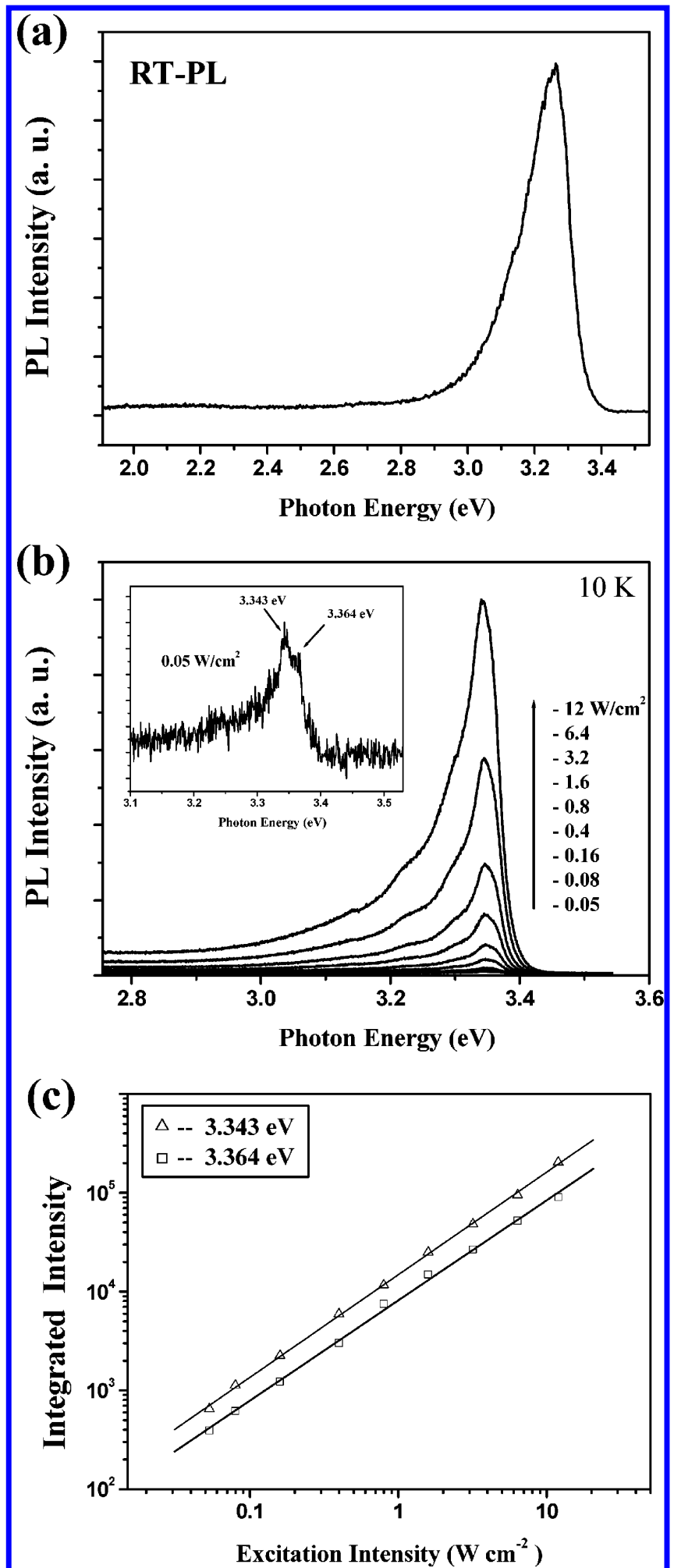

Figure 6. (a) Room-temperature photoluminescence (PL) spectrum of $\mathrm{ZnO}$ nanowires on $\mathrm{GaN}$ buffer layer. (b) PL spectra of $\mathrm{ZnO}$ nanowires as a function of excitation laser intensity (from $0.05 \mathrm{Wcm}^{-2}$ to $12 \mathrm{Wcm}^{-2}$ ) at low temperature $T=10 \mathrm{~K}$. (c) Dependence of integrated PL band intensity of $3.343 \mathrm{eV}$ and $3.364 \mathrm{eV}$ on excitation laser intensity at $T=10 \mathrm{~K}$.

the $c$-axis and suffer a small biaxial compressive stress of 121.9 $\mathrm{MPa}$. The resonant Raman spectra show good crystal quality and reveal phonon modes that are affected by the size confinement of the $\mathrm{ZnO}$ nanowire. Room-temperature and lowtemperature photoluminescence exhibit almost no nonstoichiometric defects in the crystallite. The exciton-related recombi- 
nations near the band edge dominate the UV emissions at room temperature as well as at low temperature $(T=10 \mathrm{~K})$.

Acknowledgment. All the authors would like to gratefully acknowledge partial financial support from the National Science Council (NSC) of Taiwan under Contract No. NSC-93-2112M-009-035. One of the authors, H. C. H., acknowledges NSC for providing a fellowship. We also thank the TEM group of MRL/ITRI for the help on HRTEM measurements and Dr. Wayne Lin (Bede Scientific Instruments Ltd.) for help on RSM explanation.

\section{References and Notes}

(1) Zu, P.; Tang, Z. K.; Yu, P.; Wong, G. K. L.; Kawasaki, M.; Ohtomo, A.; Koinuma, H.; Segawa, Y. Solid State Commun. 1997, 103, 459.

(2) Bagnall, D. M.; Chen, Y. F.; Zhu, Z. Q.; Yao, T.; Koyama, S.;

Shen, M. Y.; Goto, T. Appl. Phys. Lett. 1997, 70, 2230.

(3) Huang, M. H.; Wu, Y.; Feick, H.; Tran, N.; Weber, E.; Yang, P. Adv. Mater. 2001, 13, 113.

(4) Huang, M. H.; Mao, S.; Feick, H.; Yan, H.; Wu, Y.; Kind, H.; Weber, E.; Russo, R.; Yang, P. Science 2001, 292, 1897.

(5) Law, M.; Sirbuly, D.; Johnson, J.; Goldberger, J.; Saykally, R.; Yang, P. Science 2004, 305, 1269.

(6) Johnson, J. C.; Yan, H. Q.; Yang, P. D.; Saykally, R. J. J. Phys. Chem. B 2003, 107, 8816.

(7) Yan, H.; Justin, J.; Law, M.; Saykally, R.; Yang, P. Adv. Mater. 2003, 15, 1907.

(8) Johnson, J. C.; Knutsen, K. P.; Yan, H.; Law, M.; Yang, P.; Saykally, R. Nano. Lett. 2004, 4, 197.

(9) Bai, X. D.; Gao, P. X.; Wang, Z. L.; Wang, E. G. Appl. Phys. Lett.

2003, 82, 4806.

(10) Bai, X. D.; Wang, E. G.; Gao, P. X.; Wang, Z. L. Nano. Lett. 2003, 3,1147 .

(11) Kong, X. Y.; Wang, Z. L. Nano Lett. 2003, 3, 1625.

(12) Arnold, M.; Avouris, P.; Pan, Z. W.; Wang, Z. L. J. Phys. Chem. $B$ 2003, 107, 659 .

(13) Kong, X. Y.; Ding, Y.; Yang, R. S.; Wang, Z. L. Science 2004, 303,1348 .

(14) Kong, X. Y.; Wang, Z. L. Appl. Phys. Lett. 2004, 84, 975.

(15) Hughes, W. L.; Wang, Z. L. J. Am. Chem. Soc. 2004, 126, 6703.

(16) Zhao, M. H.; Wang, Z. L.; Mao, S. X. Nano Lett. 2004, 4, 587.

(17) Liu, C. H.; Zapien, J. A.; Yao, Y.; Meng, X. M.; Lee, C. S.; Fan, S. S.; Lifshitz, Y.; Lee, S. T. Adv. Mater. 2003, 15, 838.

(18) Tseng, Y. K.; Huang, C. J.; Cheng, H. M.; Lin, I. N.; Liu, K. S.; Chen, I. C. Adv. Funct. Mater. 2003, 13, 811

(19) Park, W. I.; Yi, G. C. Adv. Mater. 2004, 16, 87.

(20) Park, W. I.; Jun, Y. H.; Jung, S. W.; Yi, G. C. Appl. Phys. Lett. 2003, 82, 964.
(21) Zheng, M. J.; Zhang, L. D.; Li, G. H.; Shen, W. Z. Chem. Phys. Lett. 2002, 363, 123

(22) Park, W. I.; Kim, D. H.; Jung, S.-W.; Yi, G.-C. Appl. Phys. Lett. 2002, 80,4232

(23) Wu, J. J.; Liu, S.-C. Adv. Mater. 2002, 14, 215.

(24) Tseng, Y. K.; Chia, C. T.; Tsay, C. Y.; Lin, L. J.; Cheng, H. M.; Kwo, C. Y.; Chen, I. C. J. Electrochem. Soc. 2005, 152, G95.

(25) Ko, H. J.; Chen, Y. F.; Yao, T.; Miyajima, K.; Yamamoto, A.; Goto, T. Appl. Phys. Lett. 2000, 77, 537.

(26) Ko, H. J.; Chen, Y. F.; Hong, S. K.; Wenisch, H.; Yao, T.; Look, D. C. Appl. Phys. Lett. 2000, 77, 3761. 1511 .

(27) Nahhas, A.; Kim, H. K.; Blachere, J. Appl. Phys. Lett. 2001, 78,

(28) Vispute, R. D.; Talyansky, V.; Choopun, S.; Sharma, R. P.; Venkatesan, T.; He, M.; Tang, X.; Halpern, J. B.; Spencer, M. G.; Li, Y. X.; Salamanca-Riba, L. G.; Iliadis, A. A.; Jones, K. A. Appl. Phys. Lett. 1998, 73, 348 .

(29) Potin, V.; Ruterana, P.; Nouet, G.; Pond, R. C.; Morkoç, H. Phys. Rev. B 2000, 61, 5587.

(30) McMurdie, H.; Morris, M.; Evans, E.; Paretzkin, B.; Wong-Ng, W.; Ettlinger, L.; Hubbard, C. Powder Diffr. 1986, 1, 76.

(31) Puchert, M. K.; Timbrell, P. Y.; Lamb, R. N. J. Vac. Sci. Technol. A 1996, 14, 2220.

(32) Zhang, Y.; Jia, H.; Wang, R.; Chen, C.; Luo, X.; Yu, D.; Lee C. Appl. Phys. Lett. 2003, 83, 4631 .

(33) Damen, T. C.; Porto, S. P. S.; Tell, B. Phys. Rev. 1966, 142, 570.

(34) Scott, J. F. Phys. Rev. B 1970, 2, 1209.

(35) Zhang, X. T.; Liu, Y. C.; Zhi, Z. Z.; Zhang, J. Y.; Lu, Y. M.; Shen, D. Z.; Xu, W.; Zhong, G. Z.; Fan, X. W.; Kong, X. G. J. Phys. D 2001, 34 3430 .

(36) Ursaki, V. V.; Tiginyanu, I. M.; Zalamai, V. V.; Masalov, V. M.; Samarov, E. N.; Emelchenko, G. A.; Briones, F. J. Appl. Phys. 2004, 96, 1001.

(37) Ng, H. T.; Chen, B.; Li, J.; Han, J.; Meyyappan, M.; Wu, J.; Li, S. X.; Haller, E. E. Appl. Phys. Lett. 2003, 82, 2023.

(38) Rajalakshmi, M.; Aror, A. K.; Bendre, B. S.; Mahamuni, S. J. Appl. Phys. 2000, 87, 2445.

(39) Reynolds, D. C.; Litton, C. W.; Collins, T. C. Phys. Rev. 1965 140, A1726.

(40) Chen, Y. F.; Bagnall, D. M.; Koh, K. O.; Park, K. T.; Hiraga, K.; Zhu, Z.; Yao, T. J. Appl. Phys. 1998, 84, 3912.

(41) Chichibu, S. F.; Sota, T.; Cantwell, G.; Eason, D. B.; Litton, C. W. J. Appl. Phys. 2003, 93, 756.

(42) Jung, S. W.; Park, W. I.; Cheong, H. D.; Yi, G.-C.; Jang, H. M. Appl. Phys. Lett. 2002, 80, 1924.

(43) Hsu, H. C.; Tseng, Y. K.; Cheng, H. M.; Kuo, J. H.; Hsieh W. F. J. Cryst. Growth 2004, 261, 520. 8989. 